\title{
Kang-Xian Pills Inhibit Inflammatory Response and Decrease Gut Permeability to Treat Carbon Tetrachloride-Induced Chronic Hepatic Injury through Modulating Gut Microbiota
}

\author{
Li Wang, ${ }^{1}$ Huantian Cui, ${ }^{2,3}$ Yuting Li, ${ }^{3}$ Min Cao, ${ }^{3}$ Shanshan Man, ${ }^{1}$ Liying Guo, ${ }^{1}$ Jing Miao, \\ Jianwei Jia, ${ }^{1}$ Yuhong Bian $\mathbb{D}^{3},{ }^{3}$ and Zhaiyi Zhang $\mathbb{D}^{3}$ \\ ${ }^{1}$ Tianjin Second People's Hospital, Tianjin, China \\ ${ }^{2}$ Shandong Provincial Key Laboratory of Animal Cell and Developmental Biology, School of Life Sciences, Shandong University, \\ Qingdao, China \\ ${ }^{3}$ Tianjin University of Traditional Chinese Medicine, Tianjin, China
}

Correspondence should be addressed to Yuhong Bian; bianyuhong_2012@163.com and Zhaiyi Zhang; 3461285155@qq.com

Received 8 August 2020; Revised 24 September 2020; Accepted 5 October 2020; Published 21 October 2020

Academic Editor: Bonglee Kim

Copyright $\odot 2020$ Li Wang et al. This is an open access article distributed under the Creative Commons Attribution License, which permits unrestricted use, distribution, and reproduction in any medium, provided the original work is properly cited.

Kang-Xian (KX) pills have been clinically used for the treatment of chronic hepatic injury (CHI). However, the mechanisms of KX on CHI remain unknown. The aim of this study mainly focused on the anti-inflammatory effects of KX in a CHI mouse model based on modulating gut microbiota and gut permeability. We first established a $\mathrm{CHI}$ model using carbon tetrachloride $\left(\mathrm{CCl}_{4}\right)$ and treated it with $\mathrm{KX}$. The anti-inflammatory effects of $\mathrm{KX}$ on $\mathrm{CHI}$ model mice and the changes in gut permeability after $\mathrm{KX}$ treatment were also investigated. 16S rRNA analysis was used to study the changes of gut microbiota composition after KX treatment. In addition, gut microbiota was depleted using a combination of antibiotics in order to further confirm that KX could inhibit the inflammatory response and decrease gut permeability to treat $\mathrm{CHI}$ by modulating the gut microbiota. Results showed that KX treatment significantly improved liver function in CHI model mice. KX could also increase the levels of tight junction proteins in the colon and decrease the expression of proinflammatory cytokines in the liver. 16S rRNA analysis indicated that KX treatment affected the alpha and beta diversities in CHI model mice. Further analysis of $16 \mathrm{~S}$ rRNA sequencing indicated that KX treatment increased the ratio of Firmicutes to Bacteroidetes at the phylum level. At the genus level, KX treatment increased the relative abundance of Lactobacillus, Bacteroides, and Akkermansia and decreased the relative abundance of Ralstonia, Alloprevotella, and Lachnoclostridium. However, KX could not alleviate $\mathrm{CHI}$ after depleting the gut microbiota. The effects of KX on gut permeability and inflammatory response in the liver were also decreased following the depletion of gut microbiota. In conclusion, our current study demonstrated that gut microbiota was significantly affected during CHI progression. KX could inhibit the inflammatory response and decrease the gut permeability in $\mathrm{CHI}$ model mice through modulating the gut microbiota.

\section{Background}

Chronic hepatic injury (CHI) is an inflammatory disease that can be caused by hepatotoxicity, biological factors (hepatitis virus, bacteria, parasite, etc.), chemical factors (medicines, industrial poisons, alcohol, etc.), and environmental factors [1]. Long-term CHI can lead to hepatic fibrosis, liver cirrhosis, hepatocellular carcinoma, and liver failure [2]. Therefore, improving $\mathrm{CHI}$ is critical for preventing the occurrence of cirrhosis and liver failure.
Recent studies have shown an important role of gut microbiota dysfunction during the progression of $\mathrm{CHI}$ [3]. Carbon tetrachloride- $\left(\mathrm{CCl}_{4^{-}}\right)$induced $\mathrm{CHI}$ was exacerbated in antibiotic-treated mice as compared with mice with conventional gut microbiota [4]. The diversity and relative abundance of gut microbiota also changed in the $\mathrm{CHI}$ model [5]. Moreover, some gut microbiota species increased gut permeability and resulted in the release of bacterial metabolites, such as lipopolysaccharide (LPS), into circulation, and the increased level of LPS could contribute to the 
inflammation in liver [6]. Therefore, modulating gut microbiota is of clinical importance in the study of CHI [6].

Conventional therapies directed against $\mathrm{CHI}$ include symptomatic treatment and antibiotics. However, these drugs have side effects that include hepatotoxicity and renal toxicity, and they tend to be less effective in improving liver function [7]. Traditional Chinese medicine (TCM) has been used for the treatment of $\mathrm{CHI}$ for thousands of years [8]. Stevia has been shown to be effective in treating $\mathrm{CCl}_{4}$ induced CHI by inhibiting oxidative stress [9]. Sheep placental extract inhibits the inflammatory response in a liver injury mouse model [10]. The Taoren-Honghua herbal pair could treat $\mathrm{CHI}$ through modulating pathological microvessels and angiogenesis-associated signaling pathways [11]. San-Cao granule showed significant antiapoptotic effects on hepatic injury [12].

Kang-Xian pills (KX), produced by Tianjin Second People's Hospital, contain Angelica sinensis (Oliv.) Diels, Ligusticum wallichii Franch, Radix Scutellariae, Astragalus membranaceus, Carapax Trionycis, Radix Pseudostellariae, Szechwan Chinaberry Fruit, Schisandra chinensis, Oldenlandia diffusa, Cordyceps sinensis, Paeoniae Rubra Radix, and Glycyrrhiza uralensis and have been used for the treatment of CHI clinically. Our previous study demonstrated that oral treatment of $\mathrm{KX}(12 \mathrm{~g} / \mathrm{kg})$ for four weeks could significantly decrease the serum levels of alanine aminotransferase (ALT) and aspartate aminotransferase (AST) and increase the serum levels of albumin (ALB) and total protein (TP) in $\mathrm{CCl}_{4}$-induced $\mathrm{CHI}$ model mice. Besides, the infiltration of inflammatory cells and cellular swelling in the liver were significantly improved in KXtreated mice [13]. However, the mechanism of KX action against $\mathrm{CHI}$ remains unknown. Considering the important role of gut microbiota in liver inflammation, the aim of this study mainly focused on the anti-inflammatory effects of KX in a $\mathrm{CHI}$ mouse model based on modulating gut microbiota and gut permeability. We first established a CHI model using $\mathrm{CCl}_{4}$ and treated it with $\mathrm{KX}$. The anti-inflammatory effects of $\mathrm{KX}$ on $\mathrm{CHI}$ model mice and the changes in gut permeability after KX treatment were also investigated. $16 \mathrm{~S}$ rRNA analysis was used to study the changes in gut microbiota composition after KX treatment. Additionally, gut microbiota was depleted using a combination of antibiotics to further confirm that KX could inhibit the inflammatory response and decrease gut permeability to treat $\mathrm{CHI}$ by modulating the gut microbiota.

\section{Methods}

2.1. Reagents. Alanine aminotransferase (ALT), aspartate aminotransferase (AST), albumin (ALB), total protein (TP), and BCA test kits were purchased from Nanjing Jiancheng Biological Engineering Institute (Nanjing, China). Rabbit anti-mouse alpha-smooth muscle actin ( $\alpha$-SMA) antibody (ab7817) was purchased from Abcam (Shanghai, China). Rabbit anti-mouse tight junction protein 1 (ZO-1, 61-7300) and occludin (71-1500) antibodies were purchased from Invitrogen (USA). Mouse IL- 6 , IL- $1 \beta$, and TNF- $\alpha$ ELISA kits were obtained from Shanghai BlueGene Biotech Co., Ltd.
(Shanghai, China). Total DNA and RNA extraction kits, first-strand cDNA reverse transcription kits, polymerase chain reaction (PCR) kit, and primers were obtained from TianGen Biotechnology Co., Ltd. (Beijing, China).

2.2. Preparation of $K X$. KX pills were prepared from the pharmacy department of Tianjin Second People's Hospital. Briefly, Angelica sinensis (Oliv.) Diels (Tianjin traditional Chinese Medicine prepared pieces Co., Ltd., Tianjin, China, Batch number: 1901026), Ligusticum wallichii Franch (Tianjin traditional Chinese Medicine prepared pieces Co., Ltd., Tianjin, China, Batch number: 1812026), Radix Scutellariae (Tianjin traditional Chinese Medicine prepared pieces Co., Ltd., Tianjin, China, Batch number: 1901022), Astragalus membranaceus (Tianjin traditional Chinese Medicine prepared pieces Co., Ltd., Tianjin, China, Batch number: 1902021), Carapax Trionycis (Tianjin traditional Chinese Medicine prepared pieces Co., Ltd., Tianjin, China, Batch number: 1901012), Radix Pseudostellariae (Tianjin traditional Chinese Medicine prepared pieces Co., Ltd., Tianjin, China, Batch number: 1812005), Szechwan Chinaberry Fruit (Tianjin traditional Chinese Medicine prepared pieces Co., Ltd., Tianjin, China, Batch number: 1901023), Schisandra chinensis (Tianjin traditional Chinese Medicine prepared pieces Co., Ltd., Tianjin, China, Batch number: 1901024), Oldenlandia diffusa (Tianjin traditional Chinese Medicine prepared pieces Co., Ltd., Tianjin, China, Batch number: 1812016), Cordyceps sinensis (Tianjin traditional Chinese Medicine prepared pieces Co., Ltd., Tianjin, China, Batch number: 1812001), Paeoniae Rubra Radix (Tianjin traditional Chinese Medicine prepared pieces Co., Ltd., Tianjin, China, Batch number: 1812005), and Glycyrrhiza uralensis (Tianjin traditional Chinese Medicine prepared pieces Co., Ltd., Tianjin, China, Batch number: 1901026) were weighed according to the medical institution preparation standard in Tianjin (approval number: Z20070138). All herbs were authenticated by Pharmacist Li Wang in the Department of Pharmacy of the Tianjin Second People's Hospital. Then, herbs were crushed and sterilized for $12 \mathrm{~h}$ to obtain KX (Figure 1(a)).

2.3. Animals. Male C57BL/6 mice, weighing $20.0 \pm 2.0 \mathrm{~g}$, were obtained from Beijing Huafukang Animal Company. All animals were handled in accordance with the experimental protocols outlined by the National Institutes of Health regulation and approved by the Ethics Committee and Use Committee of the Tianjin University of Traditional Chinese Medicine.

2.4. Induction of $\mathrm{CHI}$ Using $\mathrm{CCl}_{4}$. After 3 days of acclimatization, CHI was induced as described previously [13]. In brief, the mice received intraperitoneal injections of $20 \%$ $\mathrm{CCl}_{4}$ solution diluted in olive oil $(2 \mathrm{~mL} / \mathrm{kg}$ body weight), twice weekly for a four-week period.

2.5. Depletion of the Gut Microbiota. The gut microbiota was depleted using a combination of antibiotic treatments as described previously [14]. Briefly, mice were given a 


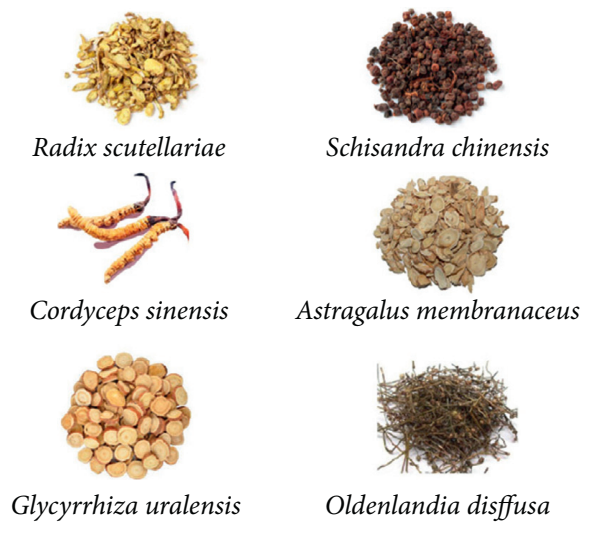

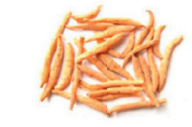

Radix pseudostellariae

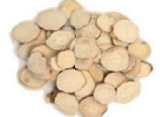

Paeoniae rubra radix

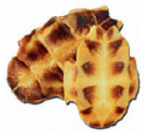

Carapax trionycis

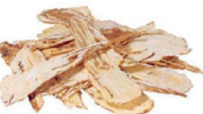

Angelica sinensis (Oliv.) Diels

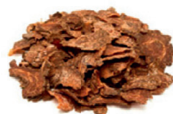

Ligusticum wallichii franch

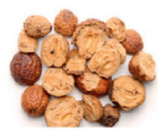

Szechwan chinaberry fruit

(a)

\begin{tabular}{|c|c|c|}
\hline $\begin{array}{c}\text { Control } \\
n=10\end{array}$ & $\begin{array}{l}\text { Intraperitoneal injection } \\
\text { of olive oil }\end{array}$ & $\begin{array}{l}\text { Oral administration of } \\
\text { normal saline }(0.2 \mathrm{~mL})\end{array}$ \\
\hline $\begin{array}{l}\text { Model } \\
n=10\end{array}$ & $\begin{array}{l}\text { Intraperitoneal injection } \\
\text { of } \mathrm{CCl}_{4} \text { to induce } \mathrm{CHI}\end{array}$ & $\begin{array}{l}\text { Oral administration of } \\
\text { normal saline }(0.2 \mathrm{~mL})\end{array}$ \\
\hline $\begin{array}{c}\mathrm{KX} \\
n=10\end{array}$ & $\begin{array}{l}\text { Intraperitoneal injection } \\
\text { of } \mathrm{CCl}_{4} \text { to induce } \mathrm{CHI}\end{array}$ & $\begin{array}{c}\text { Oral administration of } \\
\mathrm{KX}(12 \mathrm{~g} / \mathrm{kg})\end{array}$ \\
\hline
\end{tabular}

(b)

\begin{tabular}{|c|c|c|c|}
\hline $\begin{array}{c}\text { Control } \\
n=10\end{array}$ & $\begin{array}{l}\text { Received normal } \\
\text { drinking water }\end{array}$ & $\begin{array}{c}\text { Intraperitoneal injection of } \\
\text { olive oil }\end{array}$ & $\begin{array}{l}\text { Oral administration of } \\
\text { normal saline }(0.2 \mathrm{~mL})\end{array}$ \\
\hline $\begin{array}{c}\text { Antibiotics } \\
n=10\end{array}$ & $\begin{array}{c}\text { Received antibiotics } \\
\text { containing drinking water to } \\
\text { deplete the gut microbiota }\end{array}$ & $\begin{array}{l}\text { Intraperitoneal injection of } \\
\mathrm{CCl}_{4} \text { to induce } \mathrm{CHI}\end{array}$ & $\begin{array}{l}\text { Oral administration of } \\
\text { normal saline }(0.2 \mathrm{~mL})\end{array}$ \\
\hline $\begin{array}{c}\text { Antibiotics }+\mathrm{KX} \\
n=10\end{array}$ & $\begin{array}{c}\text { Received antibiotics } \\
\text { containing drinking water to } \\
\text { deplete the gut microbiota }\end{array}$ & $\begin{array}{l}\text { Intraperitoneal injection of } \\
\mathrm{CCl}_{4} \text { to induce } \mathrm{CHI}\end{array}$ & $\begin{array}{c}\text { Oral administration of } \\
\mathrm{KX}(12 \mathrm{~g} / \mathrm{kg})\end{array}$ \\
\hline
\end{tabular}

(c)

FIGURE 1: Overview of the experimental design for all groups: (a) drugs in KX; (b) mice received $\mathrm{CCl}_{4}$ injection to induce a CHI model followed by oral KX treatment; (c) mice first received a combination of antibiotics diluted in drinking water to deplete the gut microbiota. Then, mice received $\mathrm{CCl}_{4}$ injection to induce a $\mathrm{CHI}$ model followed by oral $\mathrm{KX}$ treatment.

combination of ciprofloxacin $(0.2 \mathrm{~g} / \mathrm{L})$ and metronidazole $(1 \mathrm{~g} / \mathrm{L})$ for 3 weeks in their drinking water.

2.6. Animal Grouping. Mice were randomly divided into three groups ( $n=10$ per group): Control, Model, and KX groups. The Model and $\mathrm{KX}$ groups received $\mathrm{CCl}_{4}$ injections to induce $\mathrm{CHI}$. After four weeks of $\mathrm{CCl}_{4}$ injections, mice in the Model and $\mathrm{KX}$ groups received treatment orally of either $0.2 \mathrm{~mL}$ normal saline or KX $(12 \mathrm{~g} / \mathrm{kg})$ once per day for four weeks, respectively. The Control group received an intraperitoneal injection of $0.2 \mathrm{~mL}$ olive oil twice per week for four weeks followed by oral treatment of $0.2 \mathrm{~mL}$ normal saline once per day for four weeks (Figure 1(b)).

For the gut microbiota depletion experiment, mice were randomly divided into three groups ( $n=10$ per group): Control, Antibiotics, and Antibiotics + KX. Mice in the Antibiotics and Antibiotics + KX groups were treated with a combination of antibiotics to deplete their gut microbiota followed by intraperitoneal injection of $\mathrm{CCl}_{4}$ to induce $\mathrm{CHI}$. After four weeks of $\mathrm{CCl}_{4}$ injections, mice in the Antibiotics and Antibiotics $+\mathrm{KX}$ groups received treatment orally of either $0.2 \mathrm{~mL}$ normal saline or KX $(12 \mathrm{~g} / \mathrm{kg})$ once per day for four weeks, respectively. Mice in the Control group remained untreated for three weeks followed by intraperitoneal injected of $0.2 \mathrm{~mL}$ olive oil twice per week for four weeks. After four weeks of olive oil injection, mice in the Control group received treatment orally of $0.2 \mathrm{~mL}$ normal saline once per day for four weeks (Figure 1(c)).

At the end of the four weeks of KX treatment, mice were anesthetized with ether and blood was obtained from the retrobulbar plexus for serum analysis. Then, mice were sacrificed under anesthesia, and livers and colon tissues were obtained for pathological and molecular biological studies. The liver index was calculated using the following formula: liver index $(\%)=$ liver weight $(\mathrm{g}) /$ body weight $(\mathrm{g}) \times 100$. 
2.7. Serum Biochemical Analysis. Blood samples were centrifuged at $3,000 \mathrm{rpm}$ for $15 \mathrm{~min}$ to obtain serum. The levels of ALT, AST, ALB, and TP in serum were measured by commercial test kits according to the manufacturer's protocol [13]. The absorbance value was determined using a microplate reader.

2.8. Histology. Mouse livers were fixed in paraformaldehyde after KX treatment. The liver was embedded in paraffin and subsequently cut into $5 \mu \mathrm{m}$ sections. Sections were stained with hematoxylin and eosin ( $\mathrm{H} \& \mathrm{E})$ and Masson. The ratio of collagenous fiber area to sum area in Masson staining was analyzed and quantified using ImageJ based on the integrated optical density (IOD).

2.9. Immunostaining. The expression of alpha-smooth muscle actin ( $\alpha$-SMA) in the liver and the expression of occludin and tight junction protein-1 (ZO-1) in the colon were accessed using immunostaining. The ratio of positive expressed area to sum area was analyzed and quantified using Image based on the IOD.

\subsection{Cytokine Quantification by Enzyme-Linked Immuno-} sorbent Assay (ELISA). Liver tissue ( $0.1 \mathrm{~g}$ ) was weighed and put into $900 \mathrm{~mL}$ normal saline followed by ultrasonic trituration and centrifugation at 3,000 rpm for $15 \mathrm{~min}$ to obtain tissue homogenate. The level of the total protein content in liver homogenate was detected by BCA assay according to the manufacturer's instructions (Nanjing Jiancheng Bioengineering Institute). The levels of IL- $1 \beta$, IL- 6 , and TNF- $\alpha$ in the tissue homogenate were measured using ELISA according to the manufacturer's instructions (Shanghai BlueGene Biotech Co., Ltd. China). Cytokine concentrations in liver homogenates were expressed as relative values to the total protein concentration $[15,16]$.

\subsection{RNA Isolation and Real-Time Reverse Transcription} Quantitative Polymerase Chain Reaction (qPCR). According to the manufacturer's instructions, total RNAs were isolated from the mouse livers using an RNA extraction kit. The first-strand cDNA was synthesized using $1 \mu \mathrm{g}$ of total RNA. Real-time reverse transcription-quantitative polymerase chain reaction ( $\mathrm{qPCR}$ ) was used to measure the expression of $I L-1 \beta, I L-6$, and $T N F-\alpha$ in the liver as previously described [14]. All samples were performed in triplicate and detected using a BioRad iQ5 Detection System. $\beta$-actin was used as a loading control. Quantification was performed using the $2^{-\triangle \triangle C T}$ method [17]. The sequences of the primers were listed in Table 1.

2.12. Fecal 16S rRNA Sequencing. After KX treatment, feces from the Control, Model, and KX groups were simultaneously obtained under sterile conditions in a laminar flow hood. Total DNAs were extracted from fecal samples and the $16 \mathrm{~S}$ rRNA sequencing was conducted as described previously [18].
TABle 1: Primer sequences of target genes for mice.

\begin{tabular}{lc}
\hline Genes & Primer sequence $\left(5^{\prime}-3^{\prime}\right)$ \\
\hline \multirow{2}{*}{-actin } & Forward: ACC GTG AAA AGA TGA CCC AGA \\
& Reverse: CCA GAG GCA TAC AGG GAC AA \\
$I L-1 \beta$ & Forward: ACT CAT TGT GGC TGT GGA GA \\
& Reverse: TTG TTC ATC TCG GAG CCT GT \\
$I L-6$ & Forward: AGA CTT CCA TCC AGT TGC CT \\
& Reverse: CAG GTC TGT TGG GAG TGG TA \\
TNF- $\alpha$ & Forward: ACC CTC ACA CTC ACA AACCA \\
& Reverse: GGC AGA GAG GAG GTT GAC TT \\
\hline
\end{tabular}

2.13. Statistics. All data were reported as the mean\pm standard deviation (mean \pm SD) for independent experiments. Statistical differences between the experimental groups were examined by analysis of variance (ANOVA) using SPSS version 20.0 (SPSS, Inc., Chicago, IL, USA). A $p$ value $<0.05$ was considered statistically significant. Curve fitting was carried out using the graphical package GraphPad Prism5 (GraphPad Software, Inc., La Jolla, USA).

\section{Results}

3.1. Effect of KX on CHI Mice. After four weeks of $\mathrm{KX}$ treatment, serum levels of AST, ALT, ALB, and TP were investigated to assess the therapeutic effects of $\mathrm{KX}$ on $\mathrm{CHI}$ mice. Results showed that the body weight in the Model group was lower than that in the Control group $(p<0.01)$, whereas the body weight was increased in CHI model mice after 4 weeks of KX treatment $(p<0.05$, Figure $2(a))$. The liver index was higher in the Model group compared with the Control group $(p<0.01$, Figure $2(\mathrm{~b}))$. KX treatment decreased the liver index in CHI model mice $(p<0.05$, Figure 2(b)). Additionally, the serum levels of AST and ALT were increased ( $p<0.01$, Table 2$)$, and the serum levels of ALB and TP were decreased ( $p<0.05$ and $p<0.01$, Table 2$)$ in the Model group as compared with the Control group. KX treatment decreased the serum levels of AST and ALT $(p<0.01$, Table 2$)$ and increased the serum levels of ALB and TP $(p<0.05$, Table 2$)$ in CHI model mice. Mouse livers were stained with $\mathrm{H} \& \mathrm{E}$ to observe the pathological changes after $\mathrm{KX}$ treatment. H\&E staining showed obvious infiltration of inflammatory cells and cellular swelling of hepatocytes in the Model group, whereas KX treatment ameliorated the inflammatory cell infiltration and cellular swelling of hepatocytes (Figure 2(c)). Masson staining showed an obvious deposition of collagen in the liver of CHI model mice, whereas the deposition of collagen in the liver was significantly reduced in KX-treated mice as compared to the CHI model mice $(p<0.01$, Figures 2(d) and 2(f)). Immunostaining also showed that the expression of $\alpha$-SMA in the liver was increased more in the Model group than that in the Control group $(p<0.01$, Figures 2(e) and $2(\mathrm{~g})$ ), KX treatment significantly decreased the expression of $\alpha$-SMA in the liver compared with the Model group $(p<0.01$, Figures $2(\mathrm{e})$ and $2(\mathrm{~g}))$.

3.2. Effects of KX on Gut Permeability and Levels of Proinflammatory Cytokines in the Liver. The effects of KX on gut permeability were investigated using immunostaining. Results 


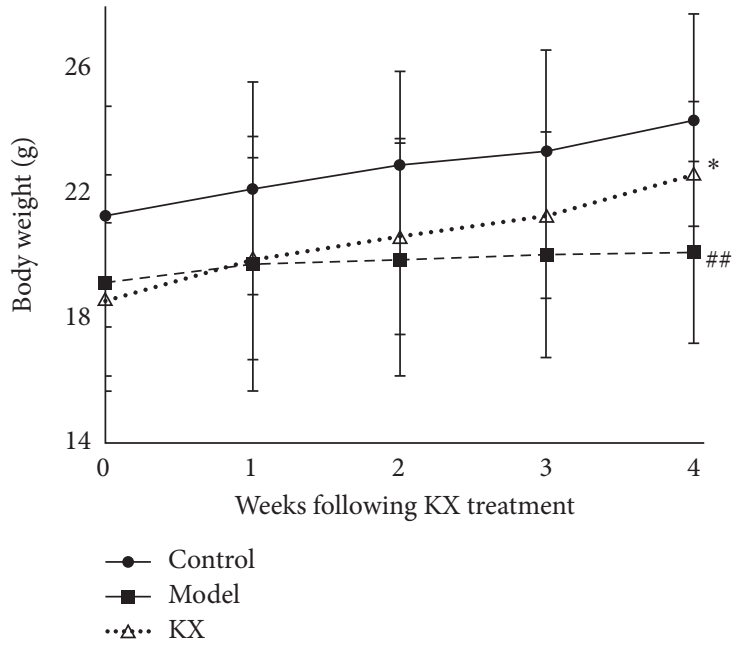

(a)

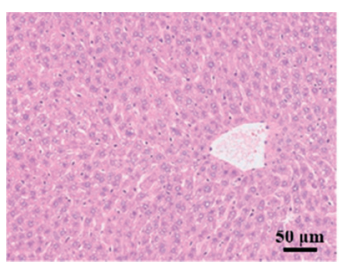

Control

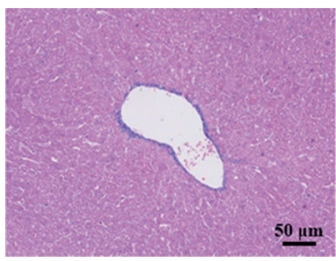

Control

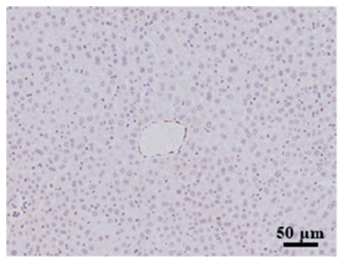

Control

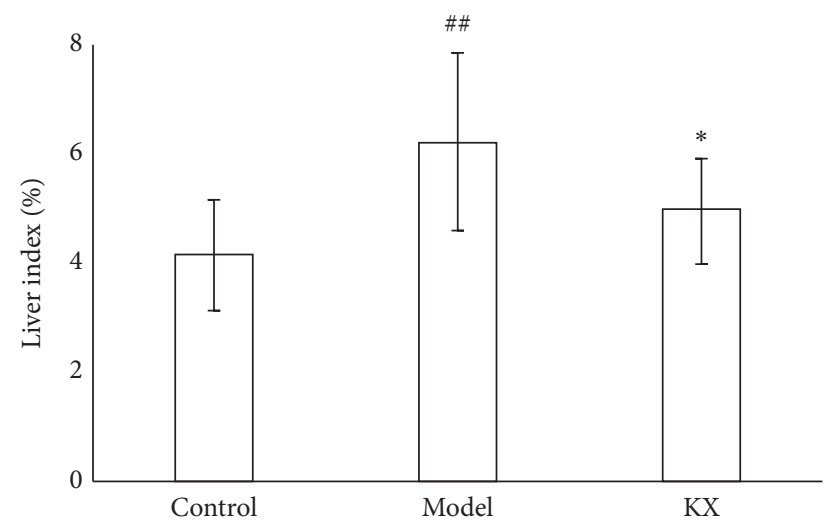

(b)

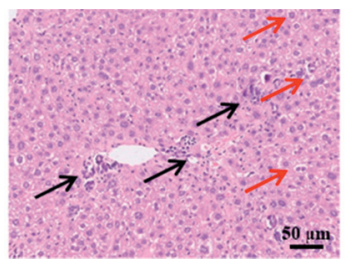

Model

(c)

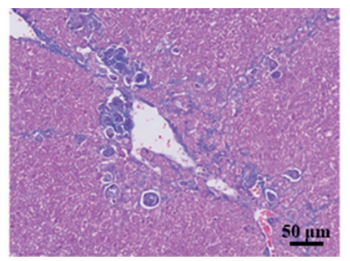

Model

(d)

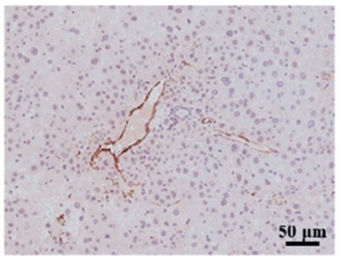

Model

(e)

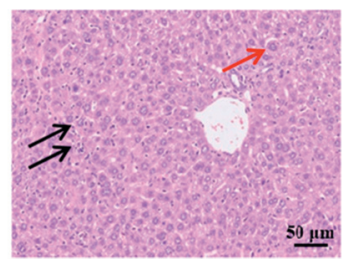

KX

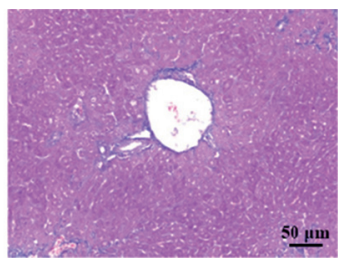

KX

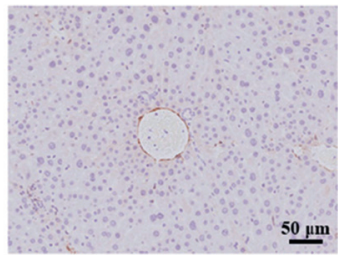

KX

FIgUre 2: Continued. 


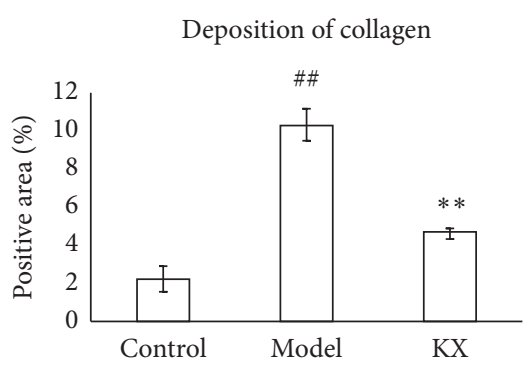

(f)

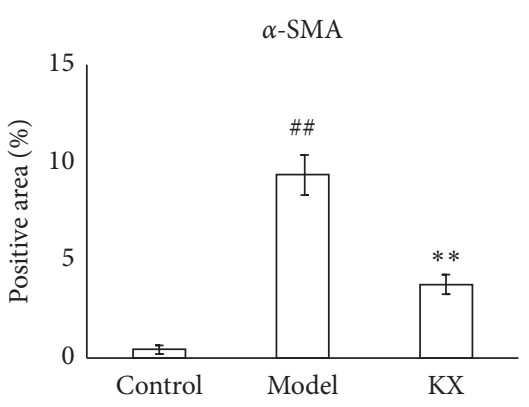

(g)

FIGURE 2: KX treatment improved the pathological changes in CHI model mice. (a, b) KX treatment increased the body weight and liver index CHI model mice. (c) H\&E staining indicated that KX treatment ameliorated the inflammatory cells infiltration and cellular swelling of hepatocytes. Black arrows indicate the inflammatory cell infiltration. Red arrows indicate the cellular swelling of hepatocytes. (d, f) Masson staining indicated that KX treatment decreased the collagen deposition in the liver. (e, g) Immunostaining indicated that KX treatment decreased the levels of $\alpha$-SMA in the liver (100x). Control, Model, and KX groups ( $n=10$ per group). Data are presented as mean \pm SD. ${ }^{\# \#} p<0.01$ compared with the Control group; ${ }^{*} p<0.05$ compared with the Model group; ${ }^{* *} p<0.01$ compared with the Model group.

TABLE 2: Concentration serum liver function biomarkers after KX treatment.

\begin{tabular}{lcccc}
\hline Groups & ALT $(\mathrm{U} / \mathrm{L})$ & AST $(\mathrm{U} / \mathrm{L})$ & ALB $(\mathrm{g} / \mathrm{L})$ & TP $(\mathrm{g} / \mathrm{L})$ \\
\hline Control & $24.58 \pm 7.27$ & $35.71 \pm 5.66$ & $42.58 \pm 4.97$ & $75.59 \pm 9.04$ \\
Model & $78.30 \pm 11.06^{\# \#}$ & $81.04 \pm 15.02^{\# \#}$ & $27.61 \pm 7.03^{\#}$ & $50.11 \pm 10.37^{\# \#}$ \\
KX & $45.41 \pm 9.31^{* *}$ & $57.92 \pm 9.88^{* *}$ & $36.52 \pm 6.95^{*}$ & $61.80 \pm 9.80^{*}$ \\
\hline
\end{tabular}

Control, Model, and KX groups ( $n=10$ per group). Data are presented as mean \pm SD. ${ }^{*}: p<0.05$ compared with the Control group; ${ }^{\# \#}: p<0.01$ compared with the Control group; ${ }^{*}: p<0.05$ compared with the Model group; ${ }^{* *}: p<0.01$ compared with the Model group.

showed that the levels of occludin $(p<0.01$, Figures $3(\mathrm{a})$ and $3(\mathrm{c}))$ and ZO-1 $(p<0.01$, Figures $3(\mathrm{~b})$ and $3(\mathrm{~d}))$ in the colon were decreased in the Model group compared with the Control group, and $\mathrm{KX}$ treatment increased the levels of occludin $(p<0.01$, Figures 3(a) and 3(c)) and ZO-1 $(p<0.01$, Figures $3(\mathrm{~b})$ and $3(\mathrm{~d}))$ in the colon. In addition, the levels and mRNA expression of IL- $1 \beta$, IL- 6 , and TNF- $\alpha$ in the liver were measured to observe the anti-inflammatory effects of $\mathrm{KX}$ on CHI. The levels of IL- $1 \beta$, IL- 6 , and TNF- $\alpha$ were significantly increased in the Model group compared with the Control group $(p<0.01$, Figure 3(e)), and KX treatment decreased the levels of IL- $1 \beta$, IL- 6 , and TNF- $\alpha$ in liver homogenate $(p<0.05, p<0.01, p<0.01$, respectively, Figure 3(e)). Likewise, the mRNA expression of $I L-1 \beta, I L-6$, and TNF- $\alpha$ was higher in the Model group $(p<0.01$, Figure 3(f)), and KX treatment downregulated the gene expression of $I L-1 \beta, I L-6$, and TNF- $\alpha$ in the liver $(p<0.05, p<0.01, p<0.05$, respectively, Figure 3(f)).

\subsection{KX Treatment Influenced the Gut Microbiota Community} in CHI Mice. High-throughput sequencing of 16S rRNA was conducted to study the changes in gut microbiota in CHI model mice after KX treatment. We obtained $1,383,228$ usable reads and 1,195 operational taxonomic units (OTUs) from 18 samples. The Shannon diversity index was higher in the Control and KX groups than that in the Model group (Figure 4(a)). Moreover, the Venn diagram revealed that 624 OTUs were common to all three groups, 896 OTUs were present in both the Control and
Model groups, 725 OTUs were present in both the Control and KX groups, and 657 OTUs were present in both the Model and KX groups (Figure 4(b)). Principal coordinates analysis (PCoA) showed a significant difference in gut microbiota in each group (Figure 4(c)). System clustering trees showed that the distance between the Control and KX groups was closer than that between the Control and Model groups (Figure 4(d)).

We further investigated the relative abundance of gut microbiota. At the phylum level, 10 phyla were found in all samples, and the most abundant phyla in all samples were Bacteroidetes and Firmicutes (Figure 4(e)). The Firmicutes to Bacteroidetes ratio ( $F$ to $B$ ratio) was significantly higher in the Model group than that in the Control group. KX treatment significantly decreased the $F$ to $B$ ratio as compared with the Model group (Figure 4(e)). At the genus level, the relative abundances of Lactobacillus and Bacteroides were lower $(p<0.05$ and $p<0.01$, respectively, Figure 4(f)) and the relative abundances of Enterococcus and Ralstonia were higher $(p<0.05$, Figure 4(f)) in the Model group than those in the Control group. However, the KX treatment increased the relative abundances of Lactobacillus and Bacteroides $(p<0.01$, Figure $4(\mathrm{f}))$ and decreased the relative abundances of Ralstonia $(p<0.01$, Figure $4(\mathrm{f}))$ in CHI model mice. In addition, $\mathrm{KX}$ treatment remarkably increased the relative abundance of Akkermansia $(p<0.05$, Figure 4(f)) and decreased the relative abundance of Alloprevotella $(p<0.01$, Figure $4(\mathrm{f}))$ and Lachnoclostridium $(p<0.05$ and $p<0.01$, respectively, Figure 4(f)) compared with both the Model and Control groups. 


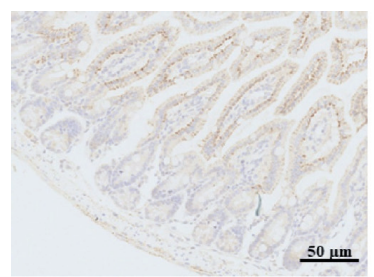

Control

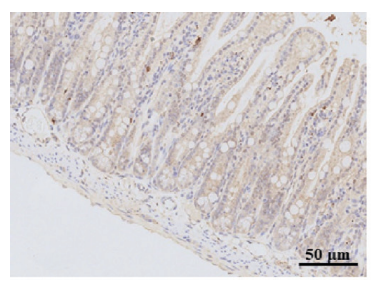

Control

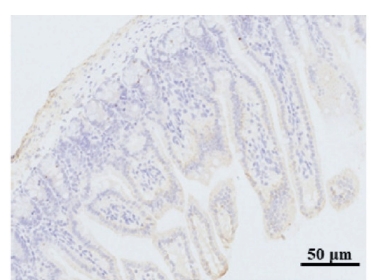

Model

(a)

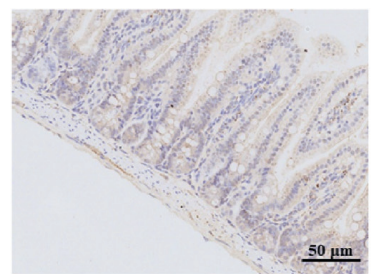

Model

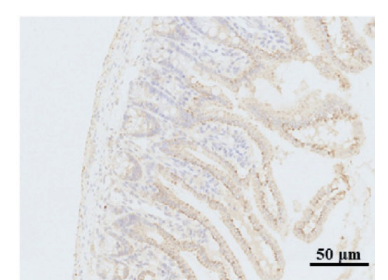

KX

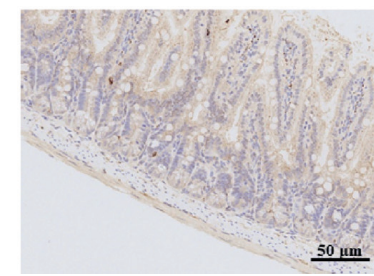

KX

(b)

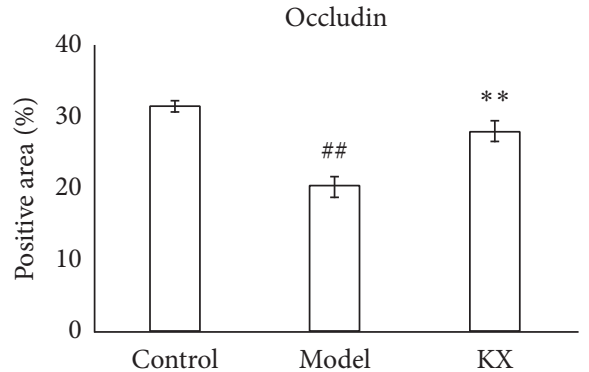

(c)

Levels of proinflammatory cytokines in liver homogenate

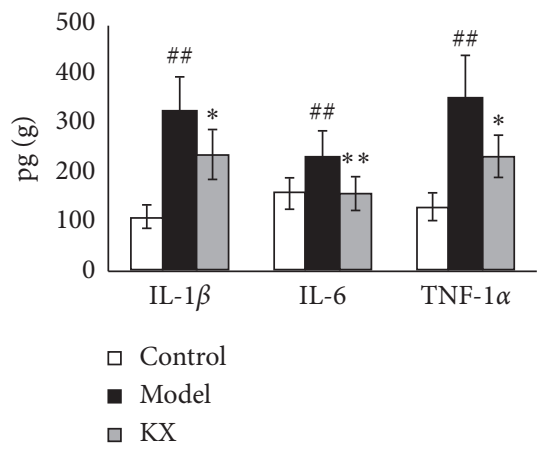

(e)

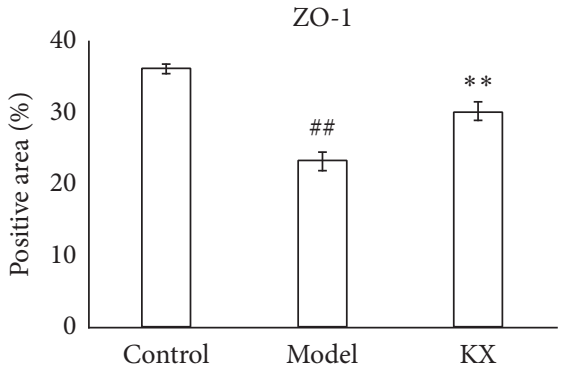

(d)

Relative mRNA expression of proinflammatory cytokines in the liver (fold to model group)

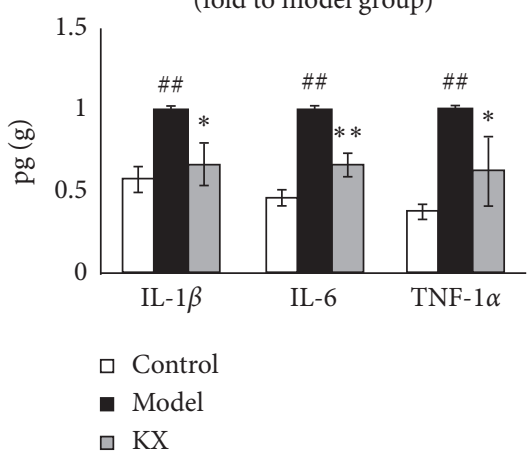

(f)

FiguRE 3: KX treatment increased gut permeability and inhibited the levels of proinflammatory cytokines in CHI model mice. Immunostaining indicated that KX treatment increased the levels of occludin (a, c) and ZO-1 (b, d) in the colon (200x). (e, f) KX treatment decreased the levels of IL- $1 \beta$, IL-6, and TNF- $\alpha$ in liver homogenate and downregulated the gene expression of $I L-1 \beta, I L-6$, and TNF- $\alpha$ in the liver. Control, Model, and KX groups ( $n=10$ per group). Data are presented as mean \pm SD. ${ }^{\# \#}: p<0.01$ compared with the Control group; ${ }^{*} p<0.05$ compared with the Model group; ${ }^{* *} p<0.01$ compared with the Model group.

3.4. Effects of KX on CHI following the Depletion of Gut Microbiota. A combination of antibiotics was used to deplete the gut microbiota followed by $\mathrm{CCl}_{4}$ and $\mathrm{KX}$ treatment to further validate KX's role in improving CHI through modulating gut microbiota. The results showed that the body weight was lower $(p<0.01$, Figure 5(a)) and the liver index $(p<0.05$, Figure 5(b)) was higher in the Antibiotics group than those in the Control group. The serum levels of AST and ALT were increased $(p<0.01$, Table 3$)$ and the serum levels of ALB and TP were decreased $(p<0.05$, Table 3 ) in the Antibiotics group as compared with the Control group. In addition, following the depletion of gut microbiota, KX treatment did not affect the body weight (not statistically significant, Figure 5(a)), liver index (not 


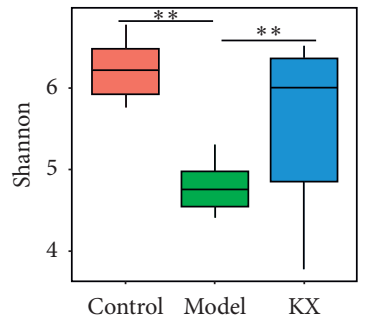

(a)

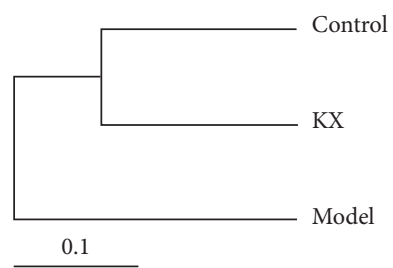

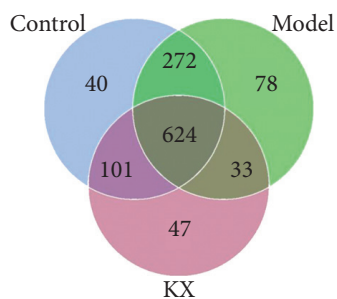

(b)

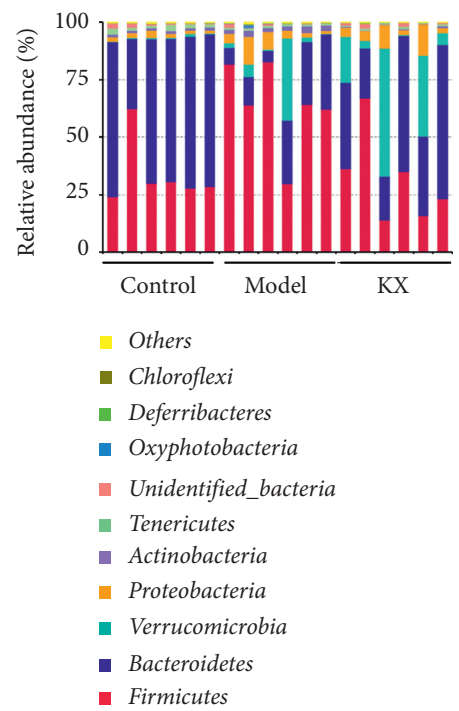

(e)

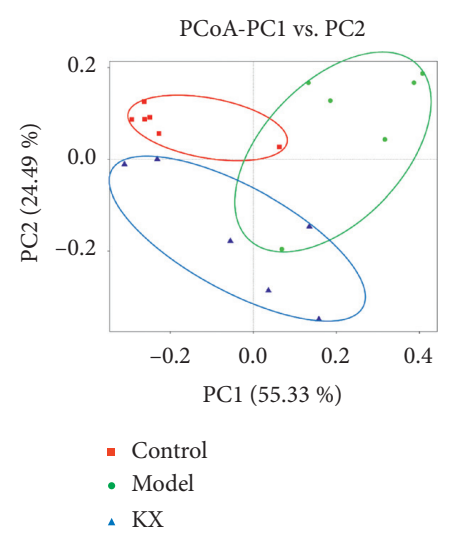

(c)

(d)

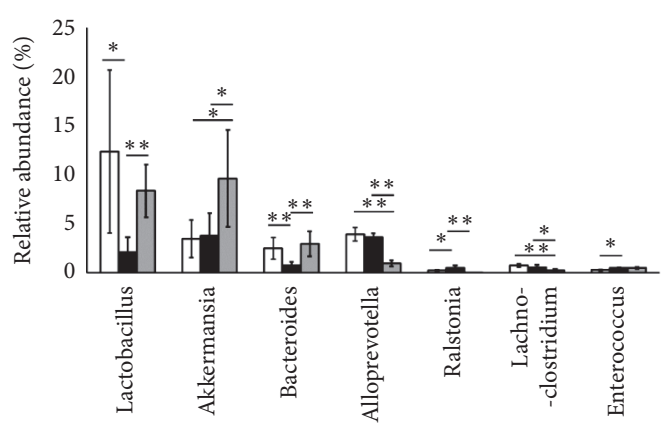

口 Control

- Model

口 KX

FIGURE 4: KX treatment changed the diversity and abundance of gut microbiota in the CHI mouse model: (a) the Shannon index was lower in the Model group as compared to the Control and KX groups; (b) the Venn diagram indicates the differential numbers of OTUs in each group; (c) PCoA score based on weighted UniFrac metrics was different in each group; (d) system clustering tree of gut microbiota based on weighted UniFrac metrics indicates the different beta diversity of gut microbiota in each group; (e) KX treatment changed the microbial community at the phylum level (bar plot); (f) at the genus level, KX treatment increased the relative abundances of Lactobacillus, Bacteroides, and Akkermansia and decreased the relative abundances of Ralstonia, Alloprevotella, and Lachnoclostridium in the CHI mouse model, the relative abundance of Enterococcus increased in the Model group, and KX treatment did not affect the relative abundance of Enterococcus in the CHI mouse model. Control, Model, and KX groups ( $n=6$ per group). Data are presented as mean \pm SD. ${ }^{*} p<0.05$; ${ }^{* *} p<0.01$.

statistically significant, Figure 5(b)), and serum levels of AST, ALT, ALB, or TP (not statistically significant, Table 3) in CHI model mice. H\&E staining showed obvious infiltration of inflammatory cells and cellular swelling of hepatocytes in the Antibiotics group, whereas there were no significant differences in inflammatory cell infiltration and cellular swelling of hepatocytes between the Antibiotics and Antibiotics + KX groups (Figure 5(c)). In addition, the Masson staining showed an obvious deposition of collagen in the Antibiotics group ( $p<0.01$, Figures 5(d) and 5(f)), whereas there were no significant differences in collagen deposition between the Antibiotics and Antibiotics + KX groups (not statistically significant, Figures 5(d) and 5(f)). Immunostaining also showed that the expression of $\alpha$-SMA in the liver was increased in the Antibiotics group than that in the Control group ( $p<0.01$, Figures 5(e) and 5(g)). There were no significant differences in $\alpha$-SMA expression between the Antibiotics and Antibiotics + KX groups (not statistically significant, Figures 5(e) and 5(g)).

3.5. Effects of KX on Gut Permeability and the Levels of Proinflammatory Cytokines in the Liver following the Depletion of Gut Microbiota. Results showed that the levels of occludin $(p<0.01$, Figures $6(\mathrm{a})$ and $6(\mathrm{c}))$ and ZO-1 $(p<0.01$, Figures 6(a) and 6(d)) in the colon were decreased in the Antibiotics group compared with the Control group. There were no significant differences in the levels of occludin (not statistically significant, Figures 6(a) and 6(c)) and ZO-1 (not statistically significant, Figure 6(a) and 6(d)) between 


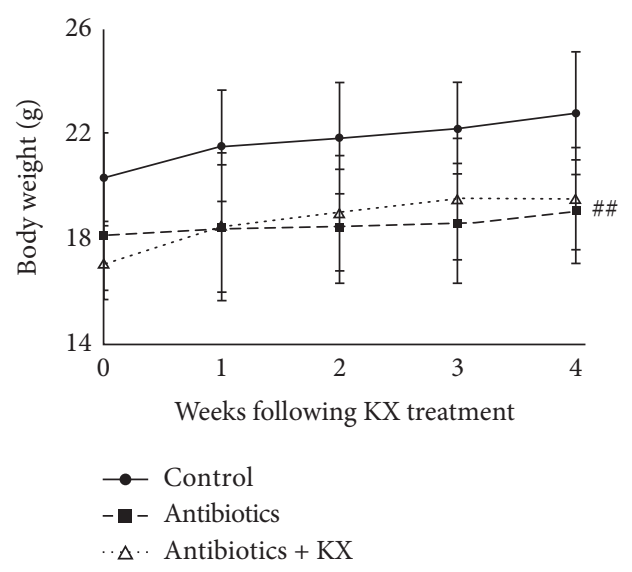

(a)

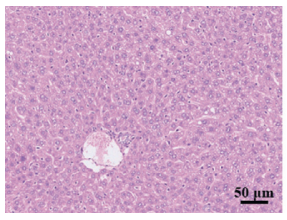

Control

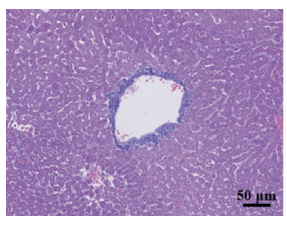

Control

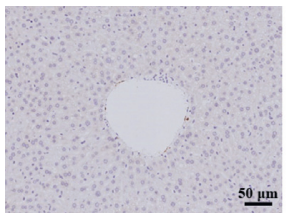

Control

Deposition of collagen

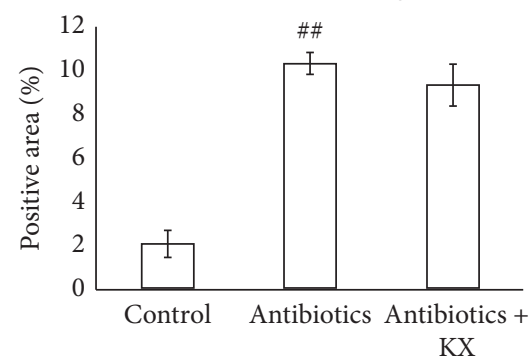

(f)

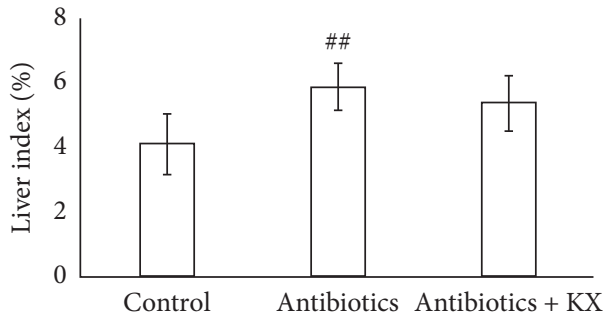

(b)

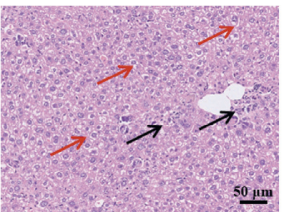

Antibiotics

(c)

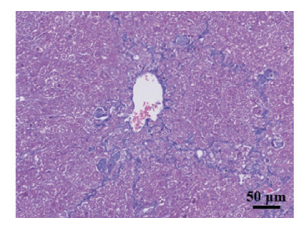

Antibiotics

(d)

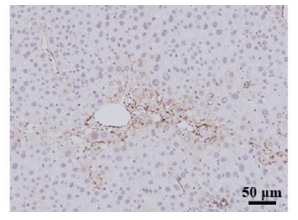

Antibiotics

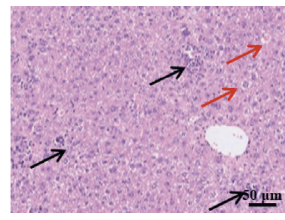

Antibiotics + KX

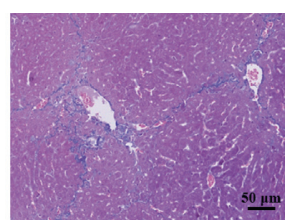

Antibiotics + KX

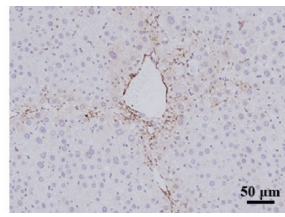

Antibiotics + KX (e)

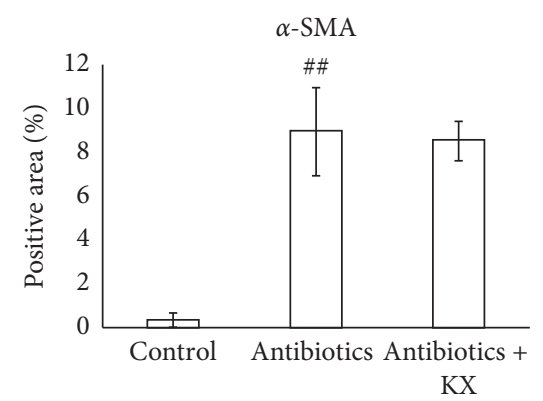

(g)

Figure 5: KX treatment did not affect the pathological changes after the depletion of gut microbiota. (a, b) KX treatment did not affect the body weight and liver index in CHI model mice after depleting the gut microbiota. (c) H\&E staining indicated that KX treatment did not affect the inflammatory cell infiltration and cellular swelling of hepatocytes after depleting the gut microbiota. Black arrows indicate the inflammatory cell infiltration. Red arrows indicate the cellular swelling of hepatocytes. (d, f) Masson staining indicated that KX treatment did not affect the deposition of collagen in the liver after depleting the gut microbiota. (e, g) Immunostaining indicated that KX treatment did not affect the level of $\alpha$-SMA in the liver after depleting the gut microbiota (100x). Control, Antibiotics, and Antibiotics + KX groups $\left(n=10\right.$ per group). Data are presented as mean \pm SD. ${ }^{\# \#} p<0.01$ compared with the Control group. 
TABLE 3: Concentration serum liver function biomarkers after antibiotics and KX treatment.

\begin{tabular}{lcccc}
\hline Groups & ALT $(\mathrm{U} / \mathrm{L})$ & AST $(\mathrm{U} / \mathrm{L})$ & ALB $(\mathrm{g} / \mathrm{L})$ & TP $(\mathrm{g} / \mathrm{L})$ \\
\hline Control & $28.51 \pm 3.27$ & $30.88 \pm 9.83$ & $38.18 \pm 2.33$ & $80.33 \pm 9.58$ \\
Antibiotics & $65.67 \pm 15.66^{\# \#}$ & $79.67 \pm 18.71^{\# \#}$ & $31.04 \pm 5.06^{\#}$ & $65.01 \pm 15.99^{\#}$ \\
Antibiotics + KX & $61.89 \pm 18.55$ & $71.85 \pm 20.46$ & $33.51 \pm 3.75$ & $59.48 \pm 12.73$ \\
\hline
\end{tabular}

Control, Antibiotics, and Antibiotics + KX groups ( $n=10$ per group). Data are presented as mean \pm SD. ${ }^{*}: p<0.05$ compared with the Control group; ${ }^{\# \#}$ : $p<0.01$ compared with the Control group.

the Antibiotics and Antibiotics $+\mathrm{KX}$ groups. In addition, the levels of IL- $1 \beta$, IL-6, and TNF- $\alpha$ were significantly increased in the Antibiotics group compared with the Control group $(p<0.01$, respectively, Figure 6(e)). There were no significant differences in the levels of IL- $1 \beta$, IL- 6 , and TNF- $\alpha$ between the Antibiotics and Antibiotics + KX groups (not statistically significant, Figure 6(e)). The mRNA expression of $I L-1 \beta, I L-6$, and TNF- $\alpha$ was higher in the Antibiotics group $(p<0.01$, respectively, Figure $6(\mathrm{f}))$. There were no significant differences in the gene expression of $I L-1 \beta, I L-6$, and TNF- $\alpha$ between the Antibiotics and Antibiotics + KX groups (not statistically significant, Figure 6(f)).

\section{Discussion}

In this study, we established an animal model of $\mathrm{CHI}$ using $\mathrm{CCl}_{4}$ injection. Our results showed that the mice in the Model group exhibited abnormal biomarkers in serum, characterized by an increase of AST and ALT and a decrease of ALB and TP. The body weight was decreased in CHI model mice. Our results also showed a significant infiltration of inflammatory cells, cellular swelling of hepatocytes, and collagen deposition in the Model group. The level of $\alpha$-SMA was also increased in the Model group. $\alpha$-SMA is highly expressed in fibrocytes. Increased level of $\alpha$-SMA has been demonstrated to be associated with the progression of liver fibrosis and liver cirrhosis. These results were in agreement with the clinical features and pathological changes in $\mathrm{CHI}$ [19]. Consistent with our previous studies, the KX treatment alleviated the abnormal serum biomarkers and pathological changes and decreased the levels of $\alpha$-SMA in CHI model mice.

Studies have shown that $\mathrm{CCL}_{4}$ could induce the release of proinflammatory cytokines such as IL- $1 \beta$, IL-6, and TNF$\alpha$ in the liver and further activate quiescent hepatic stellate cells (HSCs) and Kupffer cells [20]. The activated HSCs and Kupffer cells could in turn promote inflammatory and fibrogenic responses [21]. Decreasing the levels of IL-1 $\beta$, IL6 , and TNF- $\alpha$ could alleviate CHI and hepatic fibrosis $[15,22]$. Likewise, higher expression of proinflammatory cytokines (IL- $1 \beta$, IL- 6 , and TNF- $\alpha$ ) was observed in CHI model mice and KX treatment decreased the expression of proinflammatory cytokines in the liver. The dysfunction of gut microbiota could impair the permeability of the intestinal epithelial cell barrier and contribute to the inflammatory response in the liver [6]. Decreasing the gut permeability could alleviate the inflammatory response in the liver [23]. Our results showed that the gut permeability was increased in $\mathrm{CHI}$ model mice and KX could decrease the gut permeability through increasing the levels of ZO-1 and occludin in the colon. ZO-1 and occludin are tight junction proteins expressed in the intestinal epithelial cells [24]. Occludin could control the intestinal epithelial tight junction barrier through regulating macromolecule flux [25]. $\mathrm{ZO}-1$, also called tight junction protein 1 , is a cytoplasmic plaque protein and connects the transmembrane proteins to the cytoskeleton [26, 27].

In addition, we investigated the changes in microbiological composition using high-throughput sequencing. Our results demonstrated that the alpha diversity of the gut microbiota community was decreased in the Model group, which had a lower Shannon Diversity index than the Control group. The Shannon diversity index in the KX group was higher than the Model group, which indicated that the KX treatment increased the alpha diversity in the $\mathrm{CHI}$ mouse model. PCoA analysis revealed significant distances between all groups, indicating that the beta diversity of gut microbiota of the CHI mouse model and KX-treated mice differed from that of the Control mice. According to the system clustering tree, the beta diversity between the Control and KX groups was similar to that between the Model and Control groups. Our results also showed that the $F$ to $B$ ratio was increased in the $\mathrm{CHI}$ mouse model and was decreased after KX treatment. It has been demonstrated that the ratio of $F$ to $B$ was increased in nonalcoholic fatty liver diseases (NAFLD) [28]. However, the correlations of the $F$ to $B$ ratio in $\mathrm{CHI}$ require further study. We also found that the relative abundances of Lactobacillus, Bacteroides, and Akkermansia were increased after KX treatment. Lactobacillus is a type of probiotic and has been used to treat many liver diseases including CHI [29], NAFLD [30], alcoholic liver disease [31], and acute liver injury [32]. Oral treatment of Lactobacillus rhamnosus GG could ameliorate liver fibrosis and inflammatory response in rats [33]. The anti-inflammatory effect of $\mathrm{KX}$ on $\mathrm{CHI}$ might be through increasing the abundance of Lactobacillus in the gut. Bacteroides has been shown to produce many metabolites, such as short-chain fatty acids (SCFAs) [34] and polysaccharide A (PSA) [35]. Studies have shown that Bacteroides fragilis could protect against Bartonella henselae-induced liver damage through producing PSA [36]. SCFAs are derived from indigestible carbohydrates by the fermentation of gut microbiota and include compounds such as butyrate, propionate, and acetate [37]. Accumulated studies have reported the antiinflammatory effects of SCFAs. Butyrate could relieve colonic inflammation through inducing $\mathrm{T}$ regulatory cell (Treg) differentiation [38]. Additionally, SCFAs could suppress cholesterol synthesis in rat liver [39]. In contrast, 


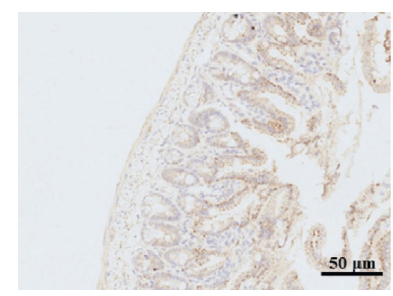

Control

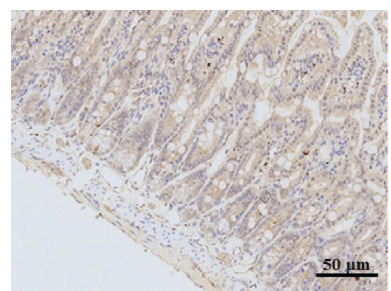

Control

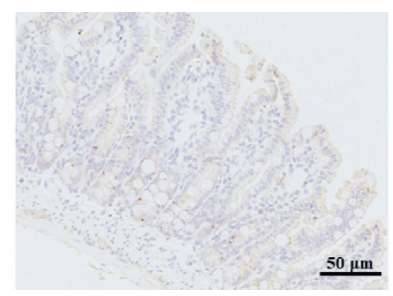

Antibiotics

(a)

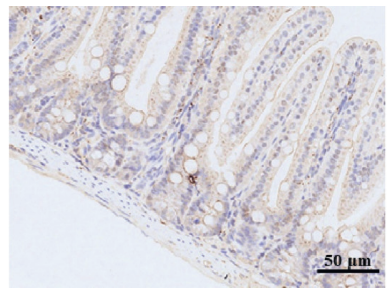

Antibiotics

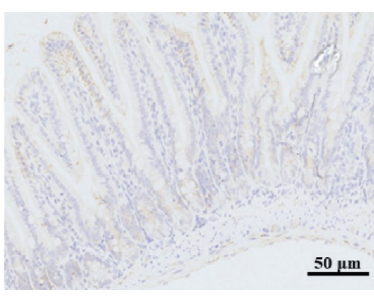

Antibiotics + KX

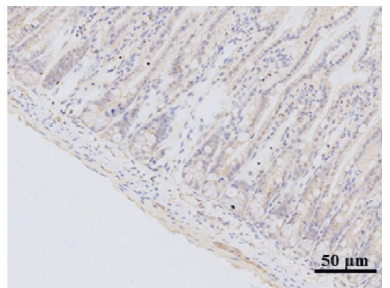

Antibiotics + KX

(b)

Occuludin

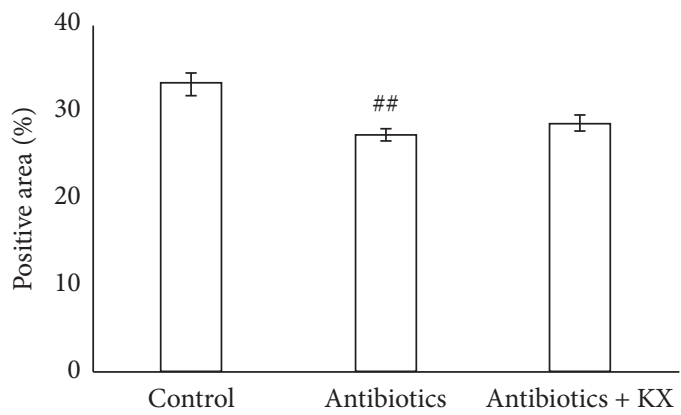

(c)

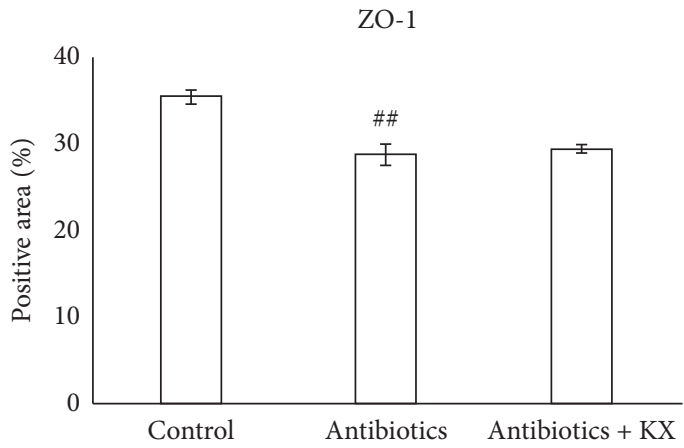

(d)

Figure 6: Continued.

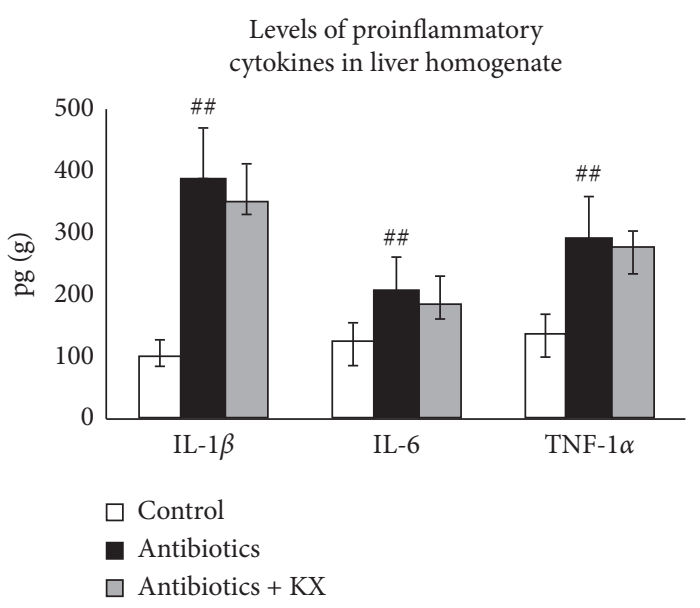

(e)
Relative mRNA expression of proinflammatory cytokines in the liver (fold to model group)

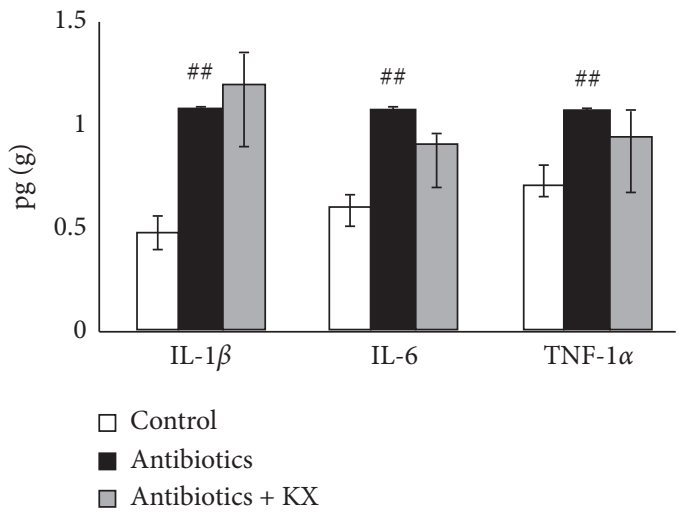

(f)

FIGURE 6: KX treatment did not affect the gut permeability and the levels of proinflammatory cytokines after depletion of gut microbiota. Immunostaining indicated that KX treatment did not affect the levels of occludin (,$c)$ and ZO-1 (b, d) in the colon after depletion of gut microbiota $(200 \times)$. (e, f) KX treatment did not affect the levels of IL-1 $\beta$, IL- 6 , and TNF- $\alpha$ in liver homogenate and the gene expression of $I L$ $1 \beta, I L-6$, and TNF- $\alpha$ in the liver after depletion of gut microbiota. Control, Antibiotics, and Antibiotics + KX groups ( $n=6$ per group). Data are presented as mean \pm SD. ${ }^{\# \#} p<0.01$ compared with the Control group. 

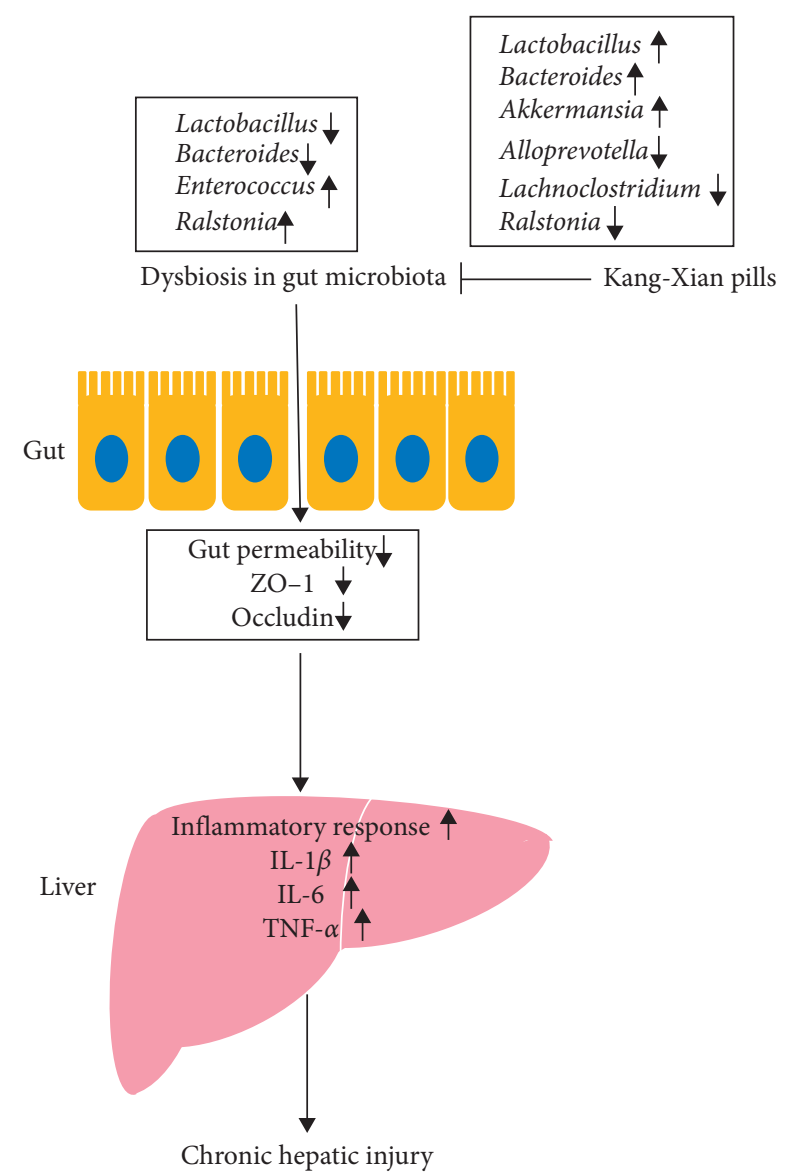

Figure 7: Graphical abstract.

studies also show that the relative abundance of Bacteroides was increased in nonalcoholic steatohepatitis (NASH) and at the end stage of hepatic fibrosis in patients [40]. Therefore, the detailed role of Bacteroides in CHI should be investigated in future studies. Akkermansia has been shown to increase the integrity of the intestine epithelial layer [41]. Moreover, Akkermansia muciniphila could prevent obesity and its associated metabolic disorders [42]. Recent studies demonstrated that Akkermansia muciniphila-derived extracellular vesicles could enhance the tight junction function on diabetic model mice. In vitro studies also showed that Akkermansia muciniphila-derived extracellular vesicles could decrease the gut permeability on human intestinal epithelial cells [43]. Increasing the abundance of Akkermansia might be the mechanism of KX on gut permeability. Our results also indicated that the relative abundance of Enterococcus was increased in the CHI mouse model. Enterococcus has been shown to cause empyema in a patient with liver disease [44]. Proton pump inhibitors could promote liver injury by inducing the overgrowth of Enterococcus [45]. Moreover, KX treatment decreased the relative abundance of Ralstonia, Alloprevotella, and Lachnoclostridium. Few studies have shown the relationship between Ralstonia, Alloprevotella, and Lachnoclostridium in CHI, which requires further studies. These results indicated that $\mathrm{KX}$ could affect the gut microbiota, decrease the gut permeability, and inhibit the inflammatory response in CHI model mice.

To further verify that KX could decrease the gut permeability and inhibit the inflammatory response to treat $\mathrm{CHI}$ through affecting gut microbiota, we used antibiotics to deplete the gut microbiota. Our previous studies demonstrated that a combination treatment of antibiotics could deplete most of the gut microbiota in mice [14], and this method did not influence the histological profiles of the liver, kidney, small intestine, or colon in mice [14]. Our current results showed that KX had a decreased effect on $\mathrm{CHI}$ following the depletion of gut microbiota, indicating that KX treatment did not alleviate $\mathrm{CHI}$ after the depletion of gut microbiota. Consistently, the effects of $\mathrm{KX}$ on gut permeability and inflammatory response in the liver were also decreased following the depletion of gut microbiota.

In conclusion, our current study demonstrated that gut microbiota was significantly affected during CHI progression. KX could inhibit the inflammatory response and decrease the gut permeability in CHI model mice through modulating the gut microbiota (Figure 7).

\section{Data Availability}

The authors declare that the datasets used and/or analyzed during the current study are available from the corresponding author on reasonable request.

\section{Conflicts of Interest}

The authors declare that they have no conflicts of interest.

\section{Authors' Contributions}

Li Wang and Huantian Cui contributed equally to this work.

\section{Acknowledgments}

This work was supported by the National Natural Science Foundation of China (81573707 and 81703921), Tianjin Municipal Commission of Health and Family Planning, Tianjin Municipal Administration of Traditional Chinese Medicine, Integrated Traditional Chinese and Western medicine Research Topics (2017073), and Science and Technology Projects in Key Fields of Traditional Chinese Medicine of Tianjin Municipal Health Commission (no. 2020006).

\section{References}

[1] A. Licata, M. G. Minissale, V. Calvaruso, and A Craxì, "A focus on epidemiology of drug-induced liver injury: analysis of a prospective cohort," European Review for Medical and Pharmacological Sciences, vol. 21, no. 1 Suppl, pp. 112-121, 2017.

[2] N. P. Chalasani, P. H. Hayashi, H. L. Bonkovsky, V. J. Navarro, W. M. Lee, and R. J. Fontana, "ACG clinical guideline: the diagnosis and management of idiosyncratic 
drug-induced liver injury," American Journal of Gastroenterology, vol. 109, no. 7, pp. 950-966, 2014.

[3] D. A. Brenner, Y.-H. Paik, and B. Schnabl, "Role of gut microbiota in liver disease," Journal of Clinical Gastroenterology, vol. 49, no. Suppl 1(1), pp. S25-S27, 2015.

[4] M. Mazagova, L. Wang, A. T. Anfora et al., "Commensal microbiota is hepatoprotective and prevents liver fibrosis in mice," The FASEB Journal, vol. 29, no. 3, pp. 1043-1055, 2014.

[5] D. E. Fouts, M. Torralba, K. E. Nelson, D. A. Brenner, and B. Schnabl, "Bacterial translocation and changes in the intestinal microbiome in mouse models of liver disease," Journal of Hepatology, vol. 56, no. 6, pp. 1283-1292, 2012.

[6] E. Seki, S. De Minicis, C. H. Österreicher et al., "TLR4 enhances TGF-03B2 signaling and hepatic fibrosis," Nature Medicine, vol. 13, no. 11, pp. 1324-1332, 2007.

[7] E. S. Björnsson, "Drug-induced liver injury due to antibiotics," Scandinavian Journal of Gastroenterology, vol. 52, no. 6-7, pp. 617-623, 2017.

[8] M. Cao, J. Miao, L. Wang, H.-Z. Liu, H.-T. Cui, and Y.-H. Bian, "The advances of traditional chinese medicine in the treatment of liver diseases in 2019," Traditional Medicine Research, vol. 5, no. 4, pp. 261-271, 2020.

[9] E. Ramos-Tovar, E. Hernández-Aquino, S. Casas-Grajales et al., "Stevia prevents acute and chronic liver injury induced by carbon tetrachloride by blocking oxidative stress through Nrf2 upregulation," Oxidative Medicine and Cellular Longevity, vol. 2018, Article ID 3823426, 12 pages, 2018.

[10] J. Liu, S. Luo, J. Yang et al., "The protective effect of sheep placental extract on concanavalin A-induced liver injury in mice," Molecules, vol. 24, no. 1, 2018.

[11] S. Xi, L. Yue, M. Shi et al., "The effects of taoren-honghua herb pair on pathological microvessel and angiogenesis-associated signaling pathway in mice model of $\mathrm{CCl}_{4}$-induced chronic liver disease," Evidence-Based Complementary and Alternative Medicine, vol. 2016, Article ID 2974256, 2016.

[12] Y. Yang, P. Zhang, Y. Wang et al., "Hepatoprotective effect of san-cao granule on con A-induced liver injury in mice and mechanisms of action exploration," Frontiers in Pharmacology, vol. 9, p. 624, 2018.

[13] H.-T. Cui, L. Wang, Y.-H. Bian, P.-D. Liu, H.-F. Zhou, and Z.-H. Pan, "Treatment of kangxian pills to chronic liver injury in mice induced with carbon tetrachloride," Chinese Herbal Medicines, vol. 7, no. 4, pp. 334-338, 2015.

[14] H. Cui, Y. Cai, L. Wang et al., "Berberine regulates treg/Th17 balance to treat ulcerative colitis through modulating the gut microbiota in the colon," Frontiers in Pharmacology, vol. 9, p. 571, 2018.

[15] S.-S. Dang, B. F. Wang, Y. A. Cheng et al., "Inhibitory effects of saikosaponin-D on $\mathrm{CCl}_{4}$-induced hepatic fibrogenesis in rats," World Journal of Gastroenterology, vol. 13, no. 4, pp. 557-563, 2007.

[16] M. Yusuke, T. Hironori, O. Satoshi et al., "Abdominal infection suppresses the number and activity of intrahepatic natural killer cells and promotes tumor growth in a murine liver metastasis model," Annals of Surgical Oncology, vol. 23, no. Suppl 2, pp. S257-S265, 2016.

[17] K. J. Livak and T. D. Schmittgen, "Analysis of relative gene expression data using real-time quantitative PCR," Methods, vol. 25, no. 4, pp. 402-408, 2002.

[18] J. Li, H. Cui, Y. Cai et al., "Tong-Xie-Yao-Fang regulates 5-HT level in diarrhea predominant irritable bowel syndrome through gut microbiota modulation," Frontiers in Pharmacology, vol. 9, p. 1110, 2018.
[19] R. Teschke and G. Danan, "Drug-induced liver injury: is chronic liver disease a risk factor and a clinical issue?" Expert Opinion on Drug Metabolism \& Toxicology, vol. 13, no. 4, pp. 425-438, 2017.

[20] E. Seki and R. F. Schwabe, "Hepatic inflammation and fibrosis: functional links and key pathways," Hepatology, vol. 61, no. 3, 2015.

[21] A. J. Czaja, "Hepatic inflammation and progressive liver fibrosis in chronic liver disease," World Journal of Gastroenterology, vol. 20, no. 10, p. 2515, 2014.

[22] M. M. Algandaby, A. M. El-Halawany, H. M. Abdallah et al., "Gingerol protects against experimental liver fibrosis in rats via suppression of pro-inflammatory and profibrogenic mediators," Naunyn-Schmiedeberg's Archives of Pharmacology, vol. 389, no. 4, pp. 419-428, 2016.

[23] F. Jing, S. Xiaoqi, X. Boyu, N. Fang, and M. Zhou, "Dahuang zexie decoction protects against high-fat diet-induced NAFLD by modulating gut microbiota-mediated toll-like receptor 4 signaling activation and loss of intestinal barrier," Evidence-Based Complementary and Alternative Medicine: Ecam, vol. 2017, no. 1, 13 pages, Article ID 2945803, 2017.

[24] J. Zhang, G. Wu, A. Shan et al., "Dietary glutamine supplementation enhances expression of ZO-1 and occludin and promotes intestinal development in Min piglets," Acta Agriculturae Scandinavica, Section A-Animal Science, vol. 67, no. 1-2, pp. 1-7, 2017.

[25] R. Al-Sadi, K. Khatib, S. Guo, D. Ye, M. Youssef, and T. Ma, "Occludin regulates macromolecule flux across the intestinal epithelial tight junction barrier," American Journal of Physiology-Gastrointestinal and Liver Physiology, vol. 300, no. 6, pp. G1054-G1064, 2011.

[26] M. Sheng and C. Sala, "PDZ domains and the organization of supramolecular complexes," Annual Review of Neuroscience, vol. 24, no. 1, p. 1, 2001.

[27] R. Ranganathan and E. M. Ross, "PDZ domain proteins: scaffolds for signaling complexes," Current Biology, vol. 7, no. 12, pp. R770-R773, 1997.

[28] M. Basaranoglu and N. Ormeci, "Nonalcoholic fatty liver disease: diagnosis, pathogenesis, and management," The Turkish Journal of Gastroenterology, vol. 25, no. 2, pp. 127-132, 2014.

[29] N. Osman, D. Adawi, S. Ahrné, B. Jeppsson, and G. Molin, "Endotoxin- and D-galactosamine-induced liver injury improved by the administration of Lactobacillus, Bifidobacterium and blueberry," Digestive and Liver Disease, vol. 39, no. 9, pp. 849-856, 2007.

[30] Y. Ritze, G. Bardos, A. Claus et al., "Lactobacillus rhamnosus GG protects against non-alcoholic fatty liver disease in mice," PLoS One, vol. 9, no. 1, Article ID e80169, 2014.

[31] A. A. Nanji, U. Khettry, and S. M. H. Sadrzadeh, "Lactobacillus feeding reduces endotoxemia and severity of experimental alcoholic liver (disease)," Experimental Biology and Medicine, vol. 205, no. 3, pp. 243-247, 1994.

[32] D. Adawi, F. B. Kasravi, G. Molin, and B. Jeppsson, "Effect ofLactobacillus supplementation with and without arginine on liver damage and bacterial translocation in an acute liver injury model in the rat," Hepatology, vol. 25, no. 3, pp. 642-647, 1997.

[33] T. O. Hammes, R. Leke, T. D. C. Escobar et al., "Lactobacillus rhamnosusGG reduces hepatic fibrosis in a model of chronic liver disease in rats," Nutrición Hospitalaria, vol. 34, no. 3, p. 702, 2017.

[34] A. Schwiertz, D. Taras, K. Schäfer et al., "Microbiota and SCFA in lean and overweight healthy subjects," Obesity, vol. 18, no. 1, pp. 190-195, 2010. 
[35] J. Ochoa-Reparaz, D. Mielcarz, L. Ditrio, A. Burroughs, S. Haque-Begum, and L. Kasper, "T.3.5. Regulatory treg cells induced by PSA-producing Bacteroides fragilis protect mice against experimental autoimmune encephalomyelitis," Clinical Immunology, vol. 131, pp. S45-S42, 2009.

[36] C. Pagliuca, A. G. Cicatiello, R. Colicchio et al., "Novel approach for evaluation of Bacteroides fragilis protective role against Bartonella henselae liver damage in immunocompromised murine model," Frontiers in Microbiology, vol. 7, p. 1750, 2016.

[37] G. T. Macfarlane and S. Macfarlane, "Bacteria, colonic fermentation, and gastrointestinal health," Journal of $A O A C$ International, vol. 95, no. 1, pp. 50-60, 2012.

[38] Y. Furusawa, Y. Obata, S. Fukuda et al., "Commensal microbederived butyrate induces the differentiation of colonic regulatory T cells," Nature, vol. 504, no. 7480, pp. 446-450, 2013.

[39] H. Hara, S. Haga, Y. Aoyama, and S. Kiriyama, "Short-chain fatty acids suppress cholesterol synthesis in rat liver and intestine," The Journal of Nutrition, vol. 129, no. 5, pp. 942-948, 1999.

[40] Z. Ling, X. Liu, Y. Cheng, and L. Li, "Predictive roles of gut dysbiosis on the severity of nonalcoholic fatty liver disease," Hepatology, vol. 64, no. 3, pp. 993-994, 2016.

[41] J. Reunanen, V. Kainulainen, L. Huuskonen et al., "Akkermansia muciniphila adheres to enterocytes and strengthens the integrity of the epithelial cell layer," Applied and Environmental Microbiology, vol. 81, no. 11, pp. 3655-3662, 2015.

[42] A. Everard, C. Belzer, L. Geurts et al., "Cross-talk between Akkermansia muciniphila and intestinal epithelium controls diet-induced obesity," Proceedings of the National Academy of Sciences, vol. 110, no. 22, pp. 9066-9071, 2013.

[43] C. Chelakkot, Y. Choi, D. K. Kim et al., "Akkermansia muciniphila-derived extracellular vesicles influence gut permeability through the regulation of tight junctions," Experimental \& Molecular Medicine, vol. 50, no. 2, Article ID e450, 2018.

[44] M. Behnia, A. S. Clay, and C. M. Hart, "Enterococcus faecalis causing empyema in a patient with liver disease," Southern Medical Journal, vol. 95, no. 10, pp. 1201-1203, 2002.

[45] C. Llorente, P. Jepsen, T. Inamine et al., "Publisher correction: gastric acid suppression promotes alcoholic liver disease by inducing overgrowth of intestinal Enterococcus," Nature Communications, vol. 8, no. 1, p. 2137, 2017. 